\title{
Fine needle aspiration cytology; is it must to be done before all thyroid surgery? Alexandria University experience
}

\author{
Galal M Abouelnagah, MD; Haytham M Awad, MD
}

Surgical Oncology Unit, Alexandria University, Egypt.

Nodular enlargement is frequent and constitutes the commonest indication for thyroidectomy. The vast majority of adult thyroid nodules are non-neoplastic lesions or benign neoplasm and fewer than 5\% are malignant. Current guidance recommends the use of fine needle aspiration cytology (FNAC) as an essential investigation in patients presenting with a thyroid lump. However, only very few studies have looked specifically at the sensitivity of FNAC in solely thyroid cancer patients. The aim of our study was to investigate the value of FNAC as a basic investigation in patients with thyroid enlargement. We aimed specifically to assess the sensitivity of FNAC within cancer suspicious group. This had been done by reviewing all cases from January 1st, 2001 to December 31st, 2012. It includes all patients with simple nodular goiter who underwent thyroid surgery during that period. Exclusion criteria: Patients who had recurrent goiter or secondary metastatic lesions. Total 617 patients were included in the study. Female to male ratio was 589 (90.6\%) and 28(9.4\%) respectively. Age of the patients ranged from 15-72 years with mean age 38.5 years. Among the 617 patients, preoperative fine needle aspiration cytology had been done to 409 cases only. Statistical analysis showed that preoperative FNAC sensitivity, specificity and accuracy are $68.27 \%, 87.40 \%$ and $85.89 \%$ respectively. We need to understand what a specific FNAC result means to patient's management. Significant result of FNAC can provide preoperative guidance when assessing the probability of the target disorder. We had expected that a "malignant" or "suggestive" FNAC result significantly enhances the probability of thyroid neoplasm and should be an absolute indication for surgery.

Key words: Goiter, preoperative assessment, indication for surgery

\section{Introduction:}

Thyroid enlargement is a common clinical finding, and it has been reported in about $4-7 \%$ of adult population. Nodular enlargement is frequent and constitutes the commonest indication for thyroidectomy. The vast majority of adult thyroid nodules are non-neoplastic lesions or benign neoplasms and fewer than $5 \%$ are malignant. ${ }^{1}$ Thyroid nodules may be cystic or solid, most of the cystic nodules are benign in nature except few cases of papillary carcinoma. Among the solid nodule only about $3 \%$ are malignant tumors. $^{2}$

The problem in clinical practice is to distinguish the few malignant thyroid nodules reliably from many benign nodules. Thyroid carcinoma closely resembles its benign counterpart in physical characteristics, measurable physiological parameters such as serum T3/T4 levels and ultrasonic characteristics. Therefore the surgical excision of the nodule and its histopathological examination is the only way to differentiate between the more benign and much less frequent malignant nodules. ${ }^{2}$ FNAC has become a common diagnostic procedure replacing other diagnostic methods as it requires no anesthesia, it is easy to perform and can be repeated without much discomfort to the patient and has relatively high diagnostic accuracy. ${ }^{2}$

Fine needle aspiration cytology (FNAC) was first used as a method of diagnosis thyroid disease by Soderstrom and Franzen in the 1950s and 1960s. Since then it has been 
accepted in many institutes. ${ }^{3}$

Current guidance recommends the use of fine needle aspiration cytology (FNAC) as an essential investigation in patients presenting with a thyroid lump. Current literature suggests that the sensitivity of FNAC in thyroid nodules ranges between $80-90 \%$. However, only very few studies have looked specifically at the sensitivity of FNAC in solely thyroid cancer patients. ${ }^{4,5}$.

Benign nodules can be caused by adenomas, colloid nodules, cysts, infectious nodules, lymphocytic or granulomatous nodules, hyperplastic nodules, thyroiditis, and congenital anomalies. Malignant nodules are classified as: 1-Differentiated: a) papillary adenocarcinoma b) follicular adenocarcinoma. 2-Medullary carcinoma. 3-Undifferentiated -a)small cell b) giant cell c) carcinosarcoma 4- Miscellaneousa) lymphoma, sarcoma b) Squamous cell carcinoma c) Fibrosarcoma d) mucoepithelial carcinoma e) metastatic tumors. ${ }^{6-8}$ Fine needle aspiration cytology (FNAC) is simple, less expensive, readily available and reliable, time saving, easy to perform, effective and almost accurate diagnostic technique for investigation of thyroid swelling. ${ }^{4,9}$ FNAC is widely accepted and has become cornerstone in evaluation of thyroid nodules because it is a simple and accurate screening test with high sensitivity and specificity in the preoperative evaluation of thyroid lesions. ${ }^{10-12}$ The role of cytology in thyroid swellings is doubled ways as it can be therapeutic, for example aspirating a large cyst can relieve pressure symptoms. Cytology also has a part to play in pre-operative diagnosis by identifying the disease process in both solitary nodules and in diffuse enlargement of the thyroid gland. ${ }^{13}$

FNAC is considered as the gold standard investigation in diagnosis of thyroid nodules. However, even FNAC has limitations because of low yield of cells, loss of histological architecture and inability to distinguish follicular adenoma and well differentiated follicular carcinoma. ${ }^{14-16}$ The aim of our study was to investigate the value of FNAC as a basic investigation in patients with thyroid enlargement. We aimed specifically to assess the sensitivity of FNAC within cancer suspicious group.

\section{Patients and methods:}

This retrospective study was carried out in the department of surgery, Alexandria Faculty of Medicine reviewing cases from January $1^{\text {st }}, 2001$ to December $31^{\text {st }}, 2012$. It includes all patients with simple nodular goiter who underwent thyroid surgery during that period. According to different policies of different units in our surgical department some patients were routinely sent for cytological evaluation of nodular goiter by FNAC and some not. All patients either had or had not FNAC were counseled about their possible diagnosis and the indications and type of surgery before operation. After thyroid surgery, the excised specimens were sent to pathology department for final histological diagnosis.

Inclusion Criteria:

1. Patients with primary euthyroid nodular goiter of any age.

2. Patients with both pre-operative FNAC and postoperative histological evaluation done by the team of pathologists in pathological department, Alexandria Faculty of Medicine.

Exclusion Criteria:

1. Patients who had recurrent goiter or secondary metastatic lesions.

2. Patients who had either pre or post operative diagnostic evaluation from any other laboratory

Statistical analysis: The data was analyzed for sensitivity, specificity and accuracy of FNAC as a predictive for thyroid surgical procedure.

\section{Results:}

In accordance with inclusion and exclusion criteria, total 617 patients were included in the study. Female to male ratio was 589 (90.6\%) and 28(9.4\%) respectively. Age of the patients ranged from 15-72 years with mean age 38.5 years. Distribution of patients in sub-groups for their age is shown in Table (1). Among the 617 patients, preoperative fine needle aspiration cytology had been done to 409 cases only.

Neoplastic lesions or suspicious of 
neoplasm had been showed in 53 of preoperative FNAC patients and out of them histopathology proved neoplastic lesions (Benign or malignant) only in $39(74 \%)$ patients, while the remaining 14 (26\%) patients were found to have non- neoplastic benign lesions. Therefore, 39 patients were found to be true positive and 14 patients were marked as false positive.

Cytological diagnosis showed nonneoplastic nodular goiter in $356(87 \%)$ patients and out of them only 15 (4\%) patients were found to have neoplastic nodular goiter on post operative histopathology. These 15 patients were marked as false negative for neoplastic nodular goiter. The major proportion 341 (96\%) patients, was found to have non-neoplastic nodular goiter on FNAC and histopathology as well. All those 341 patients were true negative for neoplasm of thyroid Table (2).

On the other hand among the 208 patients who had directly their surgery without doing preoperative FNAC only $13(6 \%)$ of them had post operative diagnosis with malignant neoplastic lesions. Of them only $8(4 \%)$ patients need completion of thyroidectomy operations as a second operation for curative surgery.

Statistical analysis showed preoperative FNAC sensitivity, specificity and accuracy are $68.27 \%, 87.40 \%$ and $85.89 \%$ respectively.

\section{Discussion:}

Thyroid nodules and goitre are common and often noted coincidentally when patients are being imaged for other reasons. The vast majority $(95 \%)$ of cases have benign disease. ${ }^{1}$

The decision whether or not to perform FNAC will depend on the clinical picture, and the responsible clinician needs to make an appropriate judgment about pursuing cytological confirmation, in order to avoid overtreatment of clinically benign conditions. ${ }^{16}$

Despite clinical size of a nodule being used as criteria in some guidelines, the evidence does not support size as a reliable indicator of malignancy. While there are some studies indicating that nodule size is associated with malignancy, a larger body of evidence suggests that size is not specific in distinguishing benign from malignant thyroid nodules. ${ }^{16}$

US appearances that are indicative of a benign nodule should be regarded as reassuring not requiring fine needle aspiration cytology (FNAC), unless the patient has a statistically high risk of malignancy. On the other hand, If the US appearances are equivocal, indeterminate or suspicious of malignancy, an US guided FNAC should follow. ${ }^{16}$

On the other hand the College of American Pathologists recommend no need for FNAC from, patients with a history of a nodule or goiter which has not changed for several years and who have no other worrying features (i.e. adult patient, no history of neck irradiation, no family history of thyroid cancer, no palpable cervical lymphadenopathy, no stridor or voice change). Also patients with a non-palpable asymptomatic nodule $<1 \mathrm{~cm}$ in diameter discovered incidentally on neck ultrasound without other worrying features are considered not indicated for FNAC. ${ }^{17}$

In nodules which are discovered incidentally; nodules under $1 \mathrm{~cm}$ should be referred to ultrasound to detect suspicious echographic characteristics that would warrant FNA. In contrast, CT and MRI are relatively insensitive for the detection of primary thyroid carcinoma, and there are little data to support FNA of nodules detected primarily through these modalities. ${ }^{18}$

In fact, any thyroid lesion with suspicious features on ultrasound should be considered for FNA, as should nodules larger than $1.5 \mathrm{~cm}$, even in the absence of suspicious features.

As a minimum, clinical information relevant to thyroid pathology should be annotated on the pathology requisition. Additional useful information includes ultrasound, CT, MRI, or nuclear imaging findings; TSH levels; concurrent thyroxine therapy; and history of prior FNA. All of these factors can influence the interpretation of a thyroid FNA because they convey situations that change expectations for a specific morphologic pattern or for the 
probability of disease. For example, low serum TSH levels are associated with a lower risk of thyroid carcinoma. ${ }^{9}$ Cellular changes that mimic malignant processes occur with I131 therapy, external beam radiation, prior FNA, autoimmune thyroiditis, and Graves' disease. ${ }^{19-21}$

According to guidelines from the American Association of Clinical Endocrinologists, FNAC is "believed to be the most effective method available for distinguishing between benign and malignant thyroid nodules, ${ }^{22}$ with an accuracy approaching 95 percent. ${ }^{6}$

In the study of Sunita and Shere et al, the correlation between fine needle aspiration cytology and the histopathological finding was high. There was $100 \%$ cyto-histopathological correlation in benign and malignant tumors of thyroid and $96.87 \%$ correlation in tumor like lesions of thyroid gland. ${ }^{1}$

Therefore, to his opinion FNAC diagnosis of malignancy is highly significant and such patients should be subjected to surgery. A benign FNAC diagnosis should be viewed with caution as false negative results do occur and these patients should be followed up and any clinical suspicion of malignancy even in the presence of benign FNAC requires surgery. ${ }^{1}$

In benign disease: in the large series of 6300 thyroid aspirates from Mayo Clinic, benign disease represented $65 \%$ of the total findings. This included thyroiditis, benign colloid as well as cystic goiter. ${ }^{23}$ On the other hand FNAC represented an improvement on the diagnosis of thyroiditis and can be considered as essential in those cases. ${ }^{24}$ This is similar to the experience of others who found that up to $63 \%$ of thyroiditis had no clinical diagnostic features. ${ }^{25}$

Similarly, in the series of Schenck, negative findings represented $81-5 \%$ of the 1000 thyroid aspirates. ${ }^{25}$ However, in these studies there is no reference to the relative contribution of the benign FNAC diagnosis to the ultimate management. In our series the FNAC, when compared with the initial clinical diagnosis based on non-invasive investigations, represented no improvement on the original diagnosis of benign colloid/ cystic goiter and can be considered as only additional to the diagnosis.

Solitary nodules which are occasionally clinically suspected and cannot be clarified by means of non-invasive investigations can be diagnosed by FNAC. In the study of Godinho, Matos et al, FNAC leads to decrease of clinically suspect lesions from $37 \%$ to $15.4 \%$ and can therefore be considered as essential in those cases. ${ }^{26}$ In the experience of other authors approximately 3-11\% of thyroid aspirates are cytologically suspicious.' This group includes diagnoses "consistent with follicular neoplasm", "consistent with Hiirthle cell neoplasm" and "suggestive of papillary carcinoma". Suspicious FNAC results require histological confirmation. In the Mayo Clinic series, $27 \%$ of the cytologically suspicious lesions proved to be malignant. ${ }^{25}$

Regarding neoplasm, out of 53 clinically suspicious cases in Godinho, Matos et al series FNAC led to diagnosis of tumor in 20 patients. However, neoplasm was not diagnosed in four cases of follicular adenoma which were cytological benign but were clinically suspicious. The false negative rate of thyroid FNAC ranges from $2 \%$ up to $33 \% .{ }^{27}$ False positive results are rare, one of 221 cases in the Mayo Clinic series. 25

In another study, false-negative rate for cytology is reported to be between 0 and $29 \%{ }^{28}$ Flanaqan et al reported that the use of one repeat FNA can increase the sensitivity for malignancy from 81.7 to $90.4 \%$ and decrease the false-negative rate from 17.1 to $11.4 \%$. Furthermore, they concluded that, with more than one repeat FNA, there was no improvement in performance characteristics. ${ }^{29}$

The management of thyroid disease as influenced by the FNAC is perhaps best exemplified in the low rate of surgical intervention. Surgery was avoided mainly in benign cystic lesions and thyroiditis as well as in anaplastic, metastatic tumours, and lymphomas. In the experience of Hamberger et al, due to the use of FNAC, only two thirds of patients with clinically solitary "cold" nodules have undergone surgery. ${ }^{30}$ The high proportion of tumors (15\%) in Godinho, 
Table 1: Distribution age \& sex among all thyroid patients treated in our institute.

\begin{tabular}{|l|l|l|l|}
\hline & Number of females & Number of males & Total number \\
\hline$<20$ & 24 & 1 & 25 \\
\hline $20-30$ & 45 & 2 & 47 \\
\hline $30-40$ & 82 & 12 & 94 \\
\hline $40-50$ & 243 & 5 & 248 \\
\hline $50-60$ & 123 & 4 & 127 \\
\hline $60-70$ & 38 & 1 & 39 \\
\hline$>70$ & 33 & 3 & 36 \\
\hline Total & 289 & 28 & 617 \\
\hline
\end{tabular}

Table 2: Relations between FNAC results \& final histological results.

\begin{tabular}{|l|l|l|l|l|l|}
\hline \multicolumn{2}{|l|}{ Patients who depends on FNAC results } & \multicolumn{2}{l|}{ Patients who depend on clinical } \\
\cline { 1 - 3 } Positive FNAC & Negative FNAC & \multicolumn{2}{l}{ assessment only } \\
\hline True positive & $39(74 \%)$ & True negative & $341(96 \%)$ & True correlation & $195(94 \%)$ \\
\hline False positive & $14(26 \%)$ & False negative & $15(4 \%)$ & False correlation & $13(6 \%)$ \\
\hline Total positive & $53(13 \%)$ & Total negative & $356(87 \%)$ & Total & 208 \\
\hline
\end{tabular}

Matos et al series is attributed to the selection of patients in a non-endemic area. In summary, they have proved FNAC to have an essential role in the diagnosis and management of $23 \%$ of their patients, a confirmatory role in $61 \%$ of patients, a non-contributory role in $13 \%$ when specimens were inadequate and a misleading role in $3 \%$ where the results were false negative. The positive identification of thyroiditis and neoplasm stands on its own as a justification for FNAC. ${ }^{27}$

At present, FNAC is a fundamental method for evaluation of suggestive thyroid nodules. Examination of the material obtained by FNAC enables clinicians to differentiate between benign and malignant processes. ${ }^{31}$ However, FNAC of thyroid nodules has its limitations. First, adequate cytological interpretation depends on correct detection of the lesion and on the aspiration technique. Wrong detection and poor aspiration techniques cause most of the false-negative reports. Second, the most important limitation of thyroid cytology lies in the differential diagnostics of cellular follicular lesions. It is especially difficult to distinguish benign from malignant follicular neoplasm by cytological evaluation because the diagnosis of cancer depends on the demonstration of vascular or capsular invasion. ${ }^{32}$ In the case of follicular lesions, the cytopathological examination relatively often concludes as follicular lesion of uncertain biological character. ${ }^{33}$

We agree with the statement that the diagnostic role of the FNAC over and above the non-invasive diagnostic procedures in benign, clinically suspicious, and malignant lesions of the thyroid can be classified into: essential, additional, and non-contributory/ misleading depending on the impact that FNAC had on the clinical management. ${ }^{26}$

From reviewing of literature and according to our results we could conclude that FNAC is not essential for each thyroid enlargement. It has a great value in preoperative assessment of suspicious neoplasm cases either clinically as single nodules or by US features. We need to understand what a specific FNAC result means to patient's management. Significant result of FNAC can provide preoperative guidance when assessing the probability of the target disorder. We had expected that a "malignant" or "suggestive" FNAC result significantly enhances the probability of thyroid neoplasm and should be an absolute indication for surgery. 


\section{Reference}

1- EA Sinna, N Ezzat: Diagnostic accuracy of fine needle aspiration cytology in thyroid lesions. Journal of the Egyptian National Cancer Institute 2012; 24: 63-70.

2- Sunita K, Anjali S, Pragati P et al: Correlation of fine needle aspiration cytology with histopathology in diagnosis of thyroid lesions. Journal of Evolution of Medical and Dental Sciences 2013; 2(26): 4826-4831.

3- Altavilla G, Pascale M, Nenci I: Fine needle aspiration cytology of thyroid gland diseases. Acta Cytol 1990; 34: 251-256.

4- Perros P, editor: Report of the Thyroid Cancer Guidelines Update Group. 2nd ed. London: Royal College of Physicians; 2007. British Thyroid Association, Royal College of Physicians. Guidelines for the management of thyroid cancer.

5- Cancer Statistics; Registrations [internet] The Office for National Statistics. [Last updated on 2005; Last cited on 2009 June 24].

6- Gharib H, Goellner JR: Fine-needle aspiration biopsy of the thyroid: An appraisal. Ann Intern Med 1993; 118: 282-289.

7- Rojeski MT, Gharib H: Nodular thyroid disease: Evaluation and management. New England J Med 1985, 313: 428.

8- Kaur K, Sonkhya N, Bapna A, Mital P: A comparative study of fine needle aspiration cytology, ultrasonography and radionucleotide scan in management of solitary thyroid nodule: A prospective analysis of 50 cases. Indian Journal of Otolaryngology and Head and Neck Surgery 2002, 54: 96.

9- Suresh Kumar, Shakil Aqil, Abdullah Dahar: Role of fine needle aspiration cytology in thyroid diseases. Journal of Surgery Pakistan (International) 2008; 13(1).

10- Arunkumar MM, Ahuja S, Chattopadhyay Tk, et al: FNAC, sonography and radionuclide scanning in solitary thyroid nodule. $J$ Asso Physicians India 1992; 40: 302.

11- Screaton NJ, Berman LH, Grant JW: USguided Core needle biopsy of the thyroid gland. Radiology 2003; 226: 827.

12- Handa U, Mohan H, Nagarkar N: Role of fine needle aspiration cytology in diagnosis and management of thyroid lesions: A study on 434 patients. J Cytol 2008; 25: 13.

13- Rajsekhara A, Sundaram C, Chowdary T, et al: Diagnostic utility of fineneedle sampling without aspiration: A prospective study. Diagn Cytopathol 1991; 7: 473-476
14- Borget I, Vielh P, Leboulleux S, et al: Assessment of the cost of FNAC as a diagnostic tool in patients with thyroid nodules. Am J Pathol 2008; 129: 763.

15- Cappelli C, Castellano M, Pirola I, et al: Thyroid nodule shape suggests malignancy. Eur J Endocrinol 2006;155: 27-31.

16- Cooper DS, Doherty GM, Haugen BR, et al: The American Thyroid Association Guidelines Taskforce. Management guidelines for patients with thyroid nodules and differentiated thyroid cancer. Thyroid 2006;16: 109-142.

17- Abati A, Sodeman TM, and the College of American Pathologists: "State of the Science" Conference for Thyroid FineNeedle Aspiration Dated Feb. 16, 2007.

18- Shetty SK, Maher MM, Hahn PF, et al: Significance of incidental thyroid lesions detected on CT: Correlation among CT, sonography, and pathology. AJR Am $J$ Roentgenol 2006; 187: 1349-1356.

19- Youserm DM, Huang T, Loevner LA, et al: Clinical and economic impact of incidental thyroid nodules found with CT and MR. AJNR Am J Neuroradiol 1997; 18: 1423-1428.

20- Are C, Hsu JF, Schoder H, et al: FDG-PET detected thyroid incidentalomas: need for further investigation?. Ann Surg Oncol 2007; 14: 239-247.

21- Chu QD, Conner MM, Lilien DL, et al: Positron emission tomography (PET) positive thyroid incidentaloma: the risk of malignancy observed in a tertiary referral center. Am Surg 2006; 72: 272-275.

22- Feld S: AACE clinical practice guidelines for the diagnosis and management of thyroid nodules. Thyroid Nodule Task Force. Endocr Pract 1996; 2: 78-84.

23- Goellner JR, Gharib H, Grant OS, Johnson DA: Fine needle cytology of the thyroid, 1980 to 1986. Acta Cytol 1987; 31: 587-590.

24- Jayaram G, Marwaha RK, Gupta RK, Sharma SK: Cytomorphologic aspects of thyroiditis: A study of 51 cases with functional, immunologic and ultrasonographic data. Acta Cytol 1987; 31: 687-693.

25- Schenk U: Zytologie der Schilddrase. Stuttgart: Springer Verlag, 1986.

26- Godinho-Matos L, Kocjan G, Kurtz A: Contribution of fine needle aspiration cytology to diagnosis and management of thyroid disease. Journal of Clinical Pathology 1992; 45: 391-395

27- Kline TS: Handbook offine needle aspiration 
biopsy cytology. St Louis: C V Mosby 1981; 153-198.

28- Hegedus L, Karstrup S: Ultrasonography in the evaluation of cold thyroid nodules. Eur $J$ Endocrinol 1998; 138(1): 30-31.

29- Kopald KH, Layfield LJ, Mohrmann R, Foshag LJ, Giuliano AE: Clarifying the role of fine-needle aspiration cytologic evaluation and frozen section in the operative management of thyroid cancer. Arch Surg 1989; 124: 1201-1205.

30- Hamberger B, Gharib H, Melton LJ, Goellner JR, Zinsmeister AR: Fine-needle aspiration biopsy of thyroid nodules: impact of thyroid practice and cost of care. Am J Med 1982; 73:
381-384.

31- Kikuchi S, Perrier ND, Ituarte PH, Treseler PA, Siperstein AE, Duh QY, et al: Accuracy of fine-needle aspiration cytology in patients with radiation-induced thyroid neoplasms. Br J Surg 2003; 90(6): 755-758.

32- Solymosi T, Toth GL, Bodo M: Diagnostic accuracy of fine needle aspiration cytology of the thyroid: impact of ultrasonography and ultrasonographically guided aspiration. Acta Cytol 2001; 45: 669-674.

33- Caplan RH, Kisken WA, Strutt PJ, Wester SM: Fine-needle aspiration biopsy of thyroid nodules, Thyroid nodule. Postgrad Med 1991; 90: 183-190. 